\title{
LA ADMINISTRACION PUBLICA EN TIEMPO DE AUSTERIDAD
}

\author{
GRUPo EUROPEO de LA AdMINISTRACIÓN PÚBLICA \\ Coloquio de Valencia, del 6 al 9 de mayo de 1987
}

El Coloquio de 1987 se ha celebrado en Valencia, organizado por el Instituto Valenciano de Administración Pública de la Generalidad Valenciana.

El tema de la conferencia era la Administración Pública en tiempo de austeridad, que ha sido examinado por seis grupos de trabajo reunidos simultáneamente para estudiar diversos aspectos. Cada uno de estos grupos estuvo animado por un director que organizaba las discusiones, teniendo en cuenta las comunicaciones presentadas por los participantes, continuarán sus trabajos en Lovaina, del 6 al 8 de septiembre del próximo año 1988.

El primer grupo, dirigido por Peter Jackson, de la Universidad de Leicester (Reino Unido), ha examinado las convergencias y divergencias de los conceptos y criterios sobre la productividad en los sectores público y privado. Su objetivo era estudiar los conceptos de productividad y rendimiento tal y como se aplican en los estudios empíricos sobre el trabajo en el sector público. Habiendo discutido sobre distintos conceptos, el grupo va a dedicarse a utilizarlos en el análisis de las prácticas seguidas en diferentes contextos con objeto de descubrir cuáles son las mejores y continuar los estudios sobre eficiencia. Para ello se cuenta con asociar de modo más directo a los trabajos del grupo a los administradores prácticos.

Lars Jeding, del Instituto Nacional para la Formación del Servicio Civil (Suecia), dirigió el segundo grupo, cuyos trabajos se referían a la adaptación de los instrumentos de medición de la productividad a la filosofía de la gestión pública y su retro-utilización por quienes adoptan las decisiones. Después de haber distinguido dos enfoques principales de la medición de la productividad, el grupo se ha pronunciado a favor de un enfoque institucional y no global $y$ ha concluido que, para la continuación de sus trabajos, el análisis de la experiencia práctica es mucho más interesante que la puesta a punto de nuevos métodos. Ha subrayado, por otra parte, que la medición de la productividad puede convertirse durante los próximos años en un importante instrumento de comunicación entre las organizaciones, los directivos y los políticos.

El tercer grupo, dirigido por Uriel Rosenthal, de la Universidad de Leyden (Holanda), tenía como tema el de cómo administrar las situaciones de crisis. El grupo se ha beneficiado de la perspectiva 
común de sus participantes sobre la noción de crisis, referida a situaciones caracterizadas por una seria amenaza, por la incertidumbre o por cierto grado de urgencia. Esta concepción común ha permitido estructurar las dimensiones en torno a una gran variedad de situaciones críticas:. desastres naturales o tecnológicos, conflictos de gran envergadura, revueltas y terrorismo. Se han examinado varios temas, como las diferencias y semejanzas nacionales respecto a la percepción, la definición y la gestión de la crisis; los procedimientos de información y comunicación; la revisión de las actuaciones comerciales; el papel de los medios de comunicación de masas; la legislación de urgencia, y el mecanismo gubernamental.

Heinrich Siedentopf, de la Escuela Superior de Ciencias Administrativas de Speyer (Alemania Federal), ha dirigido el grupo sobre las políticas de personal en tiempo de austeridad. Se ha podido llegar a algunas conclusiones generales a consecuencia de las discusiones, a pesar de las importantes diferencias que existen en Europa de un país a otro respecto a las estrategias $y$ al contexto de las restricciones y los gastos de personal. Parece que la función pública persigue actualmente dos objetivos contradictorios: por una parte, la reducción del sector público y de sus actividades, y por otra, la mejora del servicio público en su conjunto y del rendimiento individual de los funcionarios. Además, la función pública se utiliza a menudo por los gobernantes como instrumento de la política de empleo para intentar resolver los problemas de paro.

Frank Delmartino, de la Universidad Católica de Lovaina (Bélgica), y Mariano Baena del Alcázar, de la Universidad Complutense de Madrid (España), han dirigido, conjuntamente, el grupo sobre la eficacia de la Administración regional respecto a las necesidades de la población y al mejor uso de recursos limitados. Las conclusiones preliminares de este grupo indican que las regiones son instituciones político-administrativas dotadas de competencias inadecuadas sobre aspectos menores y que deben hacer frente a problemas de personal y de financiación. Hay que profundizar en estas conclusiones a partir de investigaciones empíricas sobre la productividad y la eficacia de estas instituciones, asṕ como la adecuada distribución de sus recursos financieros.

Hans Maarse, de la Universidad de Limburgo (Holanda), ha dirigido los debates del grupo sobre las autoridades locales frente a la limitación de los recursos y el aumento de las tareas. El intercambio de puntos de vista se ha referido a las cuestiones siguientes: la medida en la que el Estado central ha hecho que repercuta sobre las autoridades locales una parte desproporcio- 
nada de las restricciones; el análisis del aumento de las tareas a realizar por las autoridades locales, y el modo de adaptación de estas autoridades a la nueva situación. El grupo continuará sus actividades bajo la dirección de Barrie Houliban, del Politécnico de North Staffordshire (Reino Unido).

Con ocasión del Coloquio de Valencia se han reunido por primera vez dos grupos de estudio:

A) Grupo de estudio sobre Administración y políticas públicas en materia de agricultura, dirigido por Bernard Hoetjes, de la Universidad de Leyden (Holanda).

B) Grupo de estudio sobre La informatización en la Administración pública, presidido por Jean-Paul Baguiast, Secretario general del Comité Interministerial para la Informática y la Ofimática en la Administración (Francia).

Estos grupos se reunirán, igualmente, en Lovaina en septiembre de 1988, así como otras dos sobre La mujer en la función pública, dirigida por Margery Povall (Reino Unido), y sobre La sanidad pública, dirigida por Hans Maarse (Holanda).

El próximo Coloquio del GEAP se celebrará en 1989 y se referirá al tema «El rol de los organismos independientes titulares de potestad reglamentaria».

Puede obtenerse más información sobre el GEAP dirigiéndose al Secretariado, 1, Rue Defacqz, bte.'11, B-1050 Bruxelles. 
